\title{
Research on mechanical design of a multi-function finger rehabilitation robot
}

\author{
Yongfei Feng ${ }^{1}$, Luige Vladareanu ${ }^{2}$, Zheming Chen ${ }^{1}$, Di Jin ${ }^{1}$, Ilias Mimouni ${ }^{1}$, Hongbo Wang ${ }^{3}$ \\ ${ }^{1}$ Faculty of Mechanical Engineering \& Mechanics, Ningbo University \\ ${ }^{2}$ Robotics and Mechatronics Department, Institute of Solid Mechanics of the Romanian Academy \\ ${ }^{3}$ Parallel Robot and Mechatronic System Laboratory of Hebei Province and Key Laboratory of Advanced Forging \& Stamping \\ Technology and Science of Ministry of Education, Yanshan University
}

\section{Article Info}

Received Dec 20, 2018

\section{Keyword:}

Finger training

Rehabilitation robot

Hand physiological structure

Mechanical design

\begin{abstract}
Training with robots for injured fingers has achieved the efficacy of treatment. However, most of finger rehabilitation robots just have bending/extending movement. This paper presents a new multi-function finger rehabilitation robot with a simple mechanical structure, which could help fingers and thumb realize bending/extending movement and stretch/adduction movement. The paper firstly analyzes the hand physiological movement mechanism, confirming the motion range of each finger's joint. Based on the fingers movement rules, the robot driving structure has been developed, which includes thumb training module and fingers training module and frame. In order to prove the rationality of mechanism design, an experiment was conducted. The experiment proved that the mechanism can run smoothly, and its rope wheels also drive well without skidding phenomenon as well as its tension is appropriate.
\end{abstract}

\section{Corresponding Author:}

Luige Vladareanu, Romanian Academy, Institute of Solid Mechanics of the Romanian Academy, 15, Constantin Mille Str., District 1, Bucharest, 010141, Romania Email: luige.vladareanu@vipro.edu.ro

\section{Introduction}

\subsection{Analysis of Current Mechanical Structures of Finger Rehabilitation Robots}

Rehabilitation robotic is a new, high-tech industries, and its research application is constantly breaking through, including the innovation of the mechanical structure design and control strategy of diversification, integration, and so on ${ }^{[1-2]}$. Training with robots for injured fingers could achieve the efficacy of treatment, which also has been recognized by the clinician ${ }^{[3]}$. Different types of finger rehabilitation robot have been studied ${ }^{[4]}$. Numerous intelligent control interfaces based on advanced control strategies and human adaptive mechatronics have been developed ${ }^{[5-7]}$. The mechanical structure design of the robots can be divided into three types, 1 DOF devices, 2 DOFs devices and 3DOFs devices as shown in Table 1, where, $\mathrm{P}$ and $\mathrm{R}$ represent the sliding pair and revolve pair, respectively. P or R only has a single degree of freedom, while these two can be combined freely to be 2 DOFs devices and 3 DOFs devices as shown in Fig. 1.

Table 1. Different mechanical structure types of the finger rehabilitation robot

\begin{tabular}{cc}
\hline Number of Active Motion Pairs & Design of Composing Types \\
\hline 1 DOF Devices & P, R \\
2 DOFs Devices & RR, PR, RP, PP \\
3 DOFs Devices & RRR, RRP, RPR, PRR, RPP, PRP, PPR, PPP \\
\hline
\end{tabular}




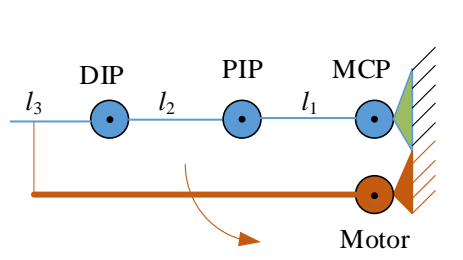

a) $1 \mathrm{DOF}$ Device

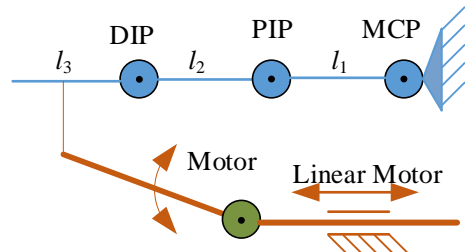

b) 2 DOFs Device

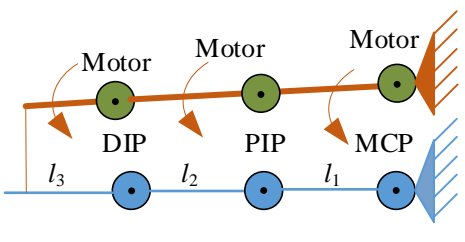

c) 3 DOFs Device

Figure 1. Example of the different mechanical structure types

As the finger bone rigid body model has 3 DOFs in bending/extending direction, the design with single DOF device can just drive the finger moving in a fixed trajectory. Refour designed a two-digit robotic exoskeleton glove mechanism, which adapts single DOF linkage mechanism to obtain bending/extending of the thumb and index finger ${ }^{[8]}$. Ma proposed a finger rehabilitation robot which is wireless, lightweight and easy to use ${ }^{[9]}$. It is realized through a serial linkages with a motor and a transmission rope. The design with 2 DOFs could help the patient realize multiple training trajectories. Lee developed an exoskeleton with two driving modules ${ }^{[10]}$. The exoskeleton will provide two forces on the middle phalange and proximal phalange, while has the functions to measure the finger joint forces and angles. 3 DOFs devices could provide joint accurate target angle training, while their control strategies are quite complicated. A 3 DOFs direct-driven serial linkage mechanism is used to be as an exoskeleton robotic system for hand rehabilitation, which provides accurate trajectories for flexing and extending movements of fingers through the independent joint control ${ }^{[11]}$. Besides, the above analysis of the finger rehabilitation robots are just considered the finger motion in bending/extending direction, and overlooked the motion in stretch/adduction direction. This paper proposed a practical finger rehabilitation robot, which could realize both bending/extending and stretch/adduction directions' motion.

\subsection{Analysis of Hand Physiological Movement Mechanism}

Hand structure is very complex, which consists of ligaments, bones, tendons, muscles and skin and soft tissue, and so on. In the process of fingers motions, their final gestures depend on the relative joint angles. As a result, the robot required movement should be determined by the joint activity, which lays the foundation for mechanical design. Each finger joints can complete bending/extending and stretch/adduction two kinds of movements. Bending/extending movement is similar to clench fist movement, and only the joints directly connected to the palm can do stretch/adduction movement. According to the finger joints motion of normal adults' activities, the joint range can be known as shown in Table 2.

Table 2. Motion range of the finger joint

\begin{tabular}{ccc}
\hline Finger Joints & Motion Mode & Range $\left(^{\circ}\right)$ \\
\hline Metacarpal-phalangeal joint (MP) & Stretch/adduction & $-20 \sim 20$ \\
Metacarpal-phalangeal joint (MP) & Bending/extending & $0 \sim 90$ \\
Proximal inter phalangeal joint (PIP) & Bending/extending & $0 \sim 110$ \\
Distal inter phalangeal joint (DIP) & Bending/extending & $0 \sim 90$ \\
\hline
\end{tabular}

\subsection{Design of Driving Structure}

Design of the driving structure is determined by the movement mode and motion range of the hand, which should simulate a variety of gestures of fingers, and provide a various joint motion ranges. PIP or DIP joints of the four fingers just do the bending/extending movement, while the MP joint maybe do the bending/extending movement. MP joint motion has no related with the PIP and DIP joints. Assuming that when one of the joints bends, the other two joints will bend; the bending/extending motion of the four fingers can be simplified to one degree of freedom, and the four fingers bends simultaneously. When the bending/extending motion of the four fingers is limited to one degree of freedom, the stretch/adduction motion of the fingers' MP joints is limited to the same plane, so the stretch/adduction motion of the four fingers can also be simplified to one degree of freedom. Ignore the thumb PIP bending movement, the thumb is simplified as two degrees of freedom of movement, including the bending/extending motion and the stretch/adduction motion. 
By analysis above, the whole hand rehabilitation exercise can be simplified into a mechanism with 4 DOFs. Thumb works on horizontal and vertical two direction. Horizontal motion is realized by mechanical and electrical slider moving on the slide rail and vertical direction with the rope transmission method, the sleeve of the thumb is fixed on the belt. Finger sleeve will move with the belt when the motor drives belt up and down. Four fingers stretch/adduction movement also uses rope transmission method and bending/extending movement is realized through the motor driving a curved sheet swinging. This meets the needs of patients hand daily activities, simplifies the driver number and compacts mechanical structure.

\section{Results and Discussion}

The finger mechanism is composed of three basic parts: thumb training module and fingers training module and frame as shown in Fig. 2. Thumb training module as shown in Fig. 3 is mainly composed of two motors, motor seat, sliding rail, pulley, nylon rope and so on. Roulette wheel 1 fixed on output shaft of 1 st motor, are connected to the pulley at the top of the thumb bracket through nylon rope, while thumb sleeve and the rope is fixed. When the 1st motor moves, rope will drive the sleeve in support of vertical guide rail moving up and down and then the thumb motion on the vertical direction can be realized, as the bending motion.

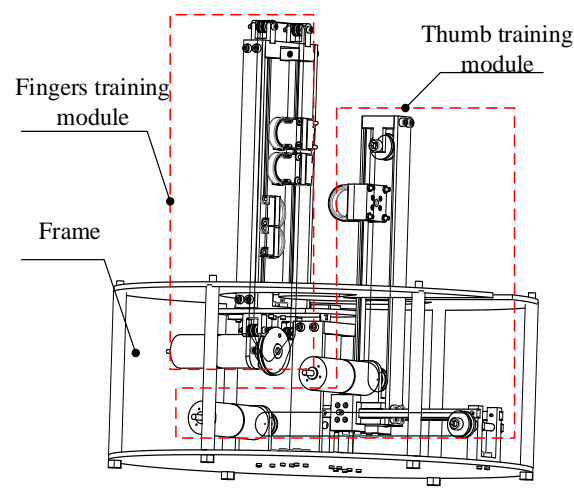

Figure 2. Structure of the whole robot

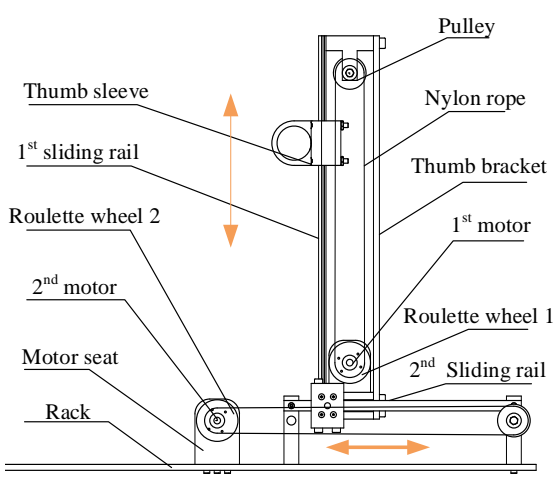

Figure 3. Structure of thumb training module

Similar with thumb bending motion, when 2 nd motor turning and reversing, nylon rope can drive thumb moving back and forth on 2 nd guide rail, thus to realize the horizontal movement of the thumb, as the stretch/adduction motion. Four fingers bracket and 4th motor bracket is fixed with the supporting plate as shown in Fig.4. When 3rd motor moves, output shaft of 3rd motor can drive the support plate in a circular trajectory, which will realize the bending/extending motion of four fingers. As the movement of each finger is different when the four fingers stretch, it is assumed four fingers can reach the maximum opening angle and the opening angle between the fingers is the same; then the moving distance of the index finger and little finger is same, so is the moving distance of the middle finger and ring finger; and the index finger's motion range is 3 times of middle finger's. So the 4th motor output shaft is installed on two concentric roulette wheel with wheel diameter ratio 3:1. Two sets of pulleys are installed on the top and bottom of the bracket. The pulleys and roulette wheel installed on the output shaft of the motor reducer constitute a rope drive mechanism. When the motor is in positive and negative rotation, the nylon rope fixed with the four finger sleeve can drive the four fingers to stretch.
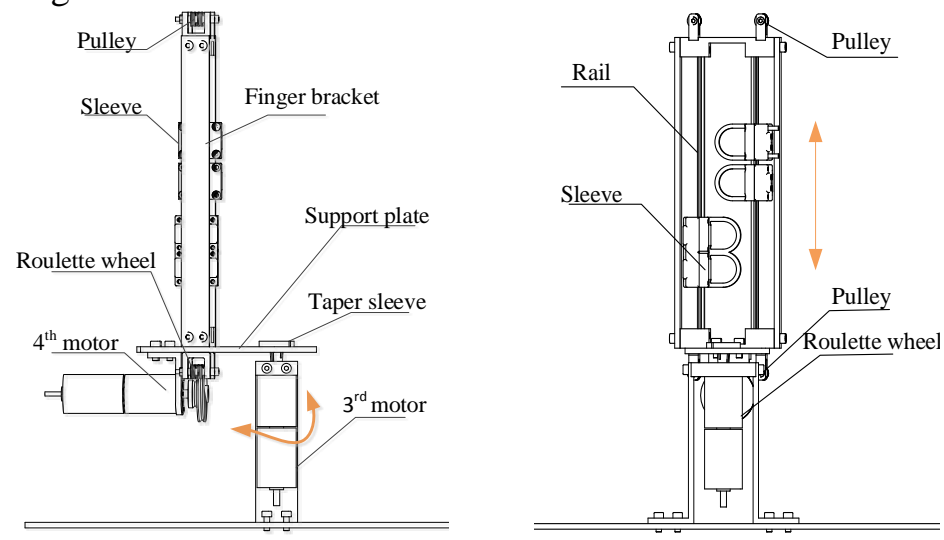

Figure 4. Mechanical structure of fingers training module 


\section{Experimental}

In order to prove the rationality of mechanism design, an experiment was conducted as shown in Fig. 5. During the whole experiment, four motors moved at the same time. When they reach the limit position at the same time, and motors' speed just turns to zero. The mechanism runs smoothly, the rope wheel can drive well without skidding phenomenon and its tension is appropriate.

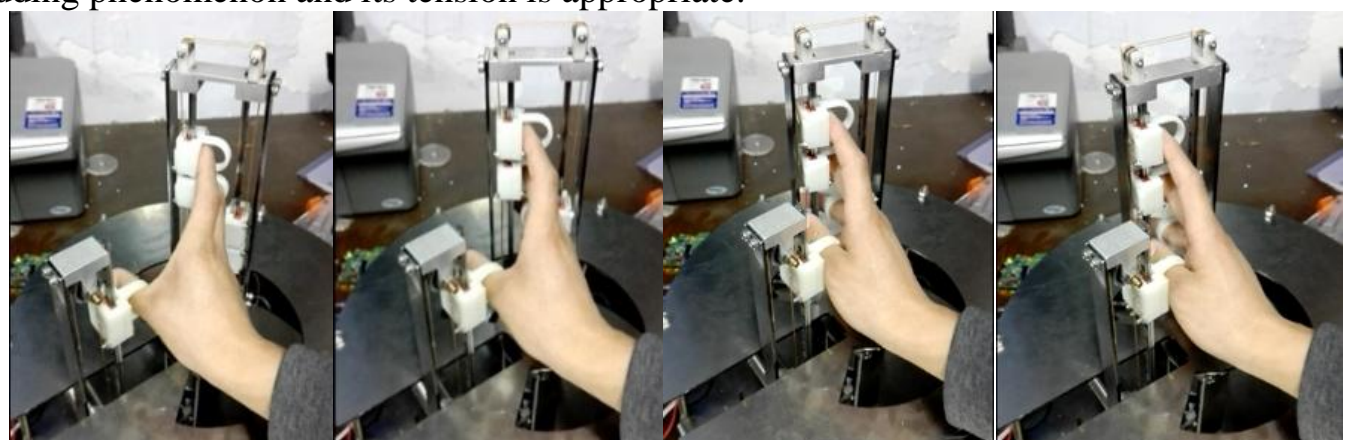

Figure 5. Training progress of the finger rehabilitation robot

\section{Conclusion}

In this paper, we design a simple mechanical structure and cost-effective training finger recovery machine. It could help fingers and thumb realize bending/extending movement and stretch/adduction movement. In the future, the intelligent control and clinical trial of the finger rehabilitation robot will be studied.

\section{Acknowledgements}

This work was supported by China Science and Technical Assistance Project for Developing Countries (KY201501009), by a grant of the Romanian Ministry of Research and Innovation, CCCDI-UEFISCDI, KEYTHROB project, number PN-III-P3-3.1-PM-RO-CN-2018-0144 / 2 BM/2018, within PNCDI III, and by the European Commission Marie Skłodowska-Curie SMOOTH project, Smart Robots for Fire-Fighting, H2020-MSCA-RISE-2016-734875.

\section{References}

[1] C. L. Jones, F.R. Wang and R. Morrison, et al, "Design and Development of the Cable Actuated Finger Exoskeleton for Hand Rehabilitation Following Stroke," IEEE-ASME Transactions on Mechatronics, vol. 19, pp. 131-140, Feb 2014.

[2] S. Jitariu, I. Staretu, "Robotized Montage Unit which Uses an Anthropomorphic Gripper with Five Fingers: CAD Modelling and Simulation", Applied Mechanics and Materials, vol. 656, pp. 146-153, Oct 2014.

[3] P. Agarwal, J. Fox and Y. Yun, et al, "An Index Finger Exoskeleton with Series Elastic Actuation for Rehabilitation: Design, Control and Performance Characterization," International Journal of Robotics Research, vol. 34, pp.1747-1772, Dec 2015.

[4] P. Polygerinos, W. Zheng and K.C. Galloway, et al, "Soft Robotic Glove for Combined Assistance and at-Home Rehabilitation," Robotics and Autonomous Systems, vol.73, pp.135-143, Nov 2017.

[5] V. Vladareanu, P. Schiopu, M.C. Deng, et al, "Intelligent Extended Control of the Walking Robot Motion," in 2014 International Conference on Advanced Mechatronic Systems, 2014, pp. 489-495.

[6] V.Vladareanu, P. Schiopu and S. Cang, et al, "Enhanced Extenics Controller for Real Time Control of Rescue Robot Actuators," in 2014 UKACC International Conference on Control, 2014, pp. 725-730.

[7] I. Staretu and C. Moldovan, "Leap Motion Device Used to Control a Real Anthropomorphic Gripper," International Journal of Advanced Robotic Systems, vol.12, pp. 1-12, Jun 2016.

[8] E. Refour, B. Sebastian and P. Ben-Tzvi, “Two-Digit Robotic Exoskeleton Glove Mechanism,” Journal of Mechanisms and Robotics-Transactions of the ASME, vol.10, pp.1-9, Apr 2017.

[9] Z. Ma and P. Ben-Tzvi, "Design and Optimization of a Five-Finger Haptic Glove Mechanism," Journal of Mechanisms and Robotics-Transactions of the ASME, vol. 7, pp.1-8, Nov 2015. 
[10] J. Lee, M. Lee, J. Bae, "Development of a Hand Exoskeleton System for Quantitative Analysis of Hand Functions". Journal of Bionic Engineering, vol. 15, pp.783-794, Sep 2018.

[11] J. Iqbal, H. Khan and N.G. Tsagarakis, "A Novel Exoskeleton Robotic System for Hand Rehabilitation Conceptualization to Prototyping," Biocybernetics and Biomedical Engineering, vol.34, pp.79-89, 2014. 